\title{
Novel rare genetic variants in idiopathic pulmonary fibrosis
}

\author{
Arnold S. Kristof (1) \\ Affiliation: Meakins-Christie Laboratories and Translational Research in Respiratory Diseases Program, \\ Research Institute of the McGill University Health Centre, Faculty of Medicine, Depts of Medicine and Critical
} Care, Montreal, QC, Canada.

Correspondence: Arnold S. Kristof, Research Institute of the McGill University Health Centre, 1001 Décarie Blvd, EM3.2219, Montreal, Quebec, Canada, H4A 3J1. E-mail: arnold.kristofamcgill.ca

@ERSpublications

The study by Nathan and co-workers on rare surfactant protein A genetic variants in patients with familial IPF illustrates the power of rare disease research consortia, and highlights the challenges in developing individualised approaches for IPF patients https://bit.ly/35uC5lw

Cite this article as: Kristof AS. Novel rare genetic variants in idiopathic pulmonary fibrosis. Eur Respir J 2020; 56: 2003252 [https://doi.org/10.1183/13993003.03252-2020].

Idiopathic pulmonary fibrosis (IPF) is a rare disease of epithelial injury leading to persistent fibrosis, remodelling of the lung parenchyma, and chronic respiratory failure [1]. IPF can be differentiated from other forms of interstitial fibrosis by the presence of radiological and histopathological features of usual interstitial pneumonia (UIP), and the absence of known initiators (e.g. occupational dusts, autoimmunity; reviewed in [2]). Despite attempts to define IPF as a uniform disease entity based on clinical and radiological criteria [3], clinicians continue to struggle with significant variability in radiological appearance, disease progression and response to interventions, which can lead to delays in diagnosis or uncertainty in patient management. This "heterogeneity" arises from genetic and environmental influences which, despite major advances in the field, have thus far been incompletely characterised. As is the case for other rare diseases, policy makers and patients have advocated for the accelerated development of individualised approaches to patient management [4,5]. Promising advances in the field include the ability to comprehensively characterise molecular and genetic features of disease using state-of-the art technologies, and orphan disease policies that facilitate the development of diagnostic and therapeutic modalities in the public and private sectors [6].

The promise of personalised medicine in IPF has led to the organisation of consortia that can effectively conduct clinical trials [7], and evaluate disease natural history and molecular pathogenesis [1]. Although the anticipated potential for precision biomarkers or designer drugs has not yet been fully realised, the research has led to important insights into mechanisms of IPF pathogenesis and heterogeneity. Genome-wide association studies identified common genetic variants associated with disease susceptibility, including those related to cellular senescence (e.g. telomere maintenance) and epithelial innate immunity (e.g. MUC5B, TOLLIP) [8]. These were complemented by studies of patients with familial IPF, in which rare variants in genes (e.g. TERT, TERC) encoding dysfunctional telomere maintenance proteins were correlated with aberrant senescence, as well as cellular responses to DNA damage and endoplasmic reticulum stress [9]. Inherited mutations in the SFTPC and SFTPA genes, encoding surfactant protein C (SP-C) and SP-A1 or 2, respectively, were linked to their abnormal cellular trafficking, proteostatic stress and pulmonary fibrosis $[8,10]$. In other rare multisystem monogenic syndromes of ageing (e.g. telomere syndromes) or vesicular trafficking (e.g. Hermansky-Pudlak syndrome), pulmonary fibrosis is a major 
manifestation [11]. The findings suggest a model in which accelerated senescence, abnormalities of proteostasis, and amplified cell stress responses in alveolar epithelial cells sensitise the distal lung epithelium to endogenous or environmental stressors. The inability to recover from injury (e.g. viral infection, smoking) leads to a persistent pro-fibrotic milieu, and the lung parenchymal remodelling typical of IPF $[1,11]$. Despite these advances, the onset and severity of IPF remain unpredictable, and familial forms tend to exhibit variable phenotypic expressivity, indicating a role for unknown genetic and epigenetic mechanisms.

The study by LEGENDRE et al. [12] published in this issue of the European Respiratory Journal provides us with a comprehensive evaluation of new SFTPA1 and SFTPA2 genetic variants, and their associated clinical manifestations. Representing the French National Network for Rare Lung Diseases (RespiFIL), they describe the largest such cohort thus far published in the literature, identifying 11 heterozygous mutations inherited by eight affected individuals and eight of their family members in autosomal dominant fashion. The mutations encode substitutions of conserved amino acid residues in the carbohydrate recognition domain (CRD) of SP-A1 or SP-A2. By computational modelling, the mutations did not appear to affect global protein structure, although this approach does not reflect the potential contribution of native post-translational modifications or protein-protein interactions. When engineered in recombinant proteins and expressed in HEK293T cells, the mutations led to cellular retention of SP-A1 or SP-A2. Available tissue samples exhibited increased levels of SP-A1 or SP-A2 in hyperplastic epithelial cells. The intracellular retention and cytotoxic effects of a SP-A2 CRD mutant, as well as those related to SP-C mutations, were previously described $[10,13]$.

The study by LEgEndre et al. [12] also highlights several pervasive challenges in the field. Despite the robust effect of all SFTPA1 and SFTPA2 mutations on secretion of their encoded proteins, there was significant variability in disease onset, progression and radiological appearance. In fact, similar limitations in genotype/phenotype correlation have been observed in other forms of familial IPF and rare diseases of autosomal dominant inheritance. Potential mechanisms include incomplete penetrance, additional inherited genetic modifiers, or environmental agents acting via epigenetic modifications. For single dominant mutations, incomplete penetrance has been explained in model organisms (e.g. C. elegans) by inter-individual differences in the expression levels or function of encoded mRNA and/or protein [14]. This intra-generational variability may be due to differences in the activity of other genes in the same signalling network, epigenetic modifiers of gene expression (e.g. transcriptional co-regulators, non-coding miRNAs), or protein degradation or trafficking. Although the levels of SP-A protein or mRNA were not evaluated in the study by LEGENDRE et al. [12], evolving technologies that interrogate the whole genome, transcriptome and epigenome, will permit the evaluation of monogenic defects in the context of the individual's full genetic and epigenetic background. Some of these new technologies (e.g. detection of DNA methylation, bulk and single cell RNA sequencing) have already been applied in large cohorts of patients with sporadic IPF. Analyses of these population-based datasets are gradually clarifying how genetic and environmental factors modify disease progression, and how to better define patient endotypes (i.e. subsets of patients classified by common biological mechanisms) with distinct prognostic risk and responses to therapies $[15,16]$.

The lack of correlation between SP-A1 or -2 secretion defects and clinical phenotype may also be due to the use of a preclinical model (i.e. expression of recombinant proteins in HEK293T cells) that does not fully reproduce the native molecular and genomic environment. Emerging technologies, however, are providing new opportunities to establish more individualised prognostic biomarkers. For instance, induced pluripotent stem cells can be engineered from patient samples, permitting the evaluation of disease associated variants in the patient's own genetic background. In addition, gene editing technologies (e.g. CRISPR-Cas9) can circumvent the need for recombinant protein expression in the evaluation of genetic variants.

Despite their variable expressivity, detection of the described SFTPA1 and SFTPA2 variants provides important and actionable information for physicians and patients. When consensus criteria are applied [17], the variants can be considered pathogenic, and their detection signals heightened surveillance for clinically apparent fibrosis and lung cancer in carriers, as well as the potential risk to offspring. Conversely, those relatives without the pathogenic variant might be reassured, given their low likelihood of developing disease. A role for fetal SFTPA variant detection in prenatal screening would appear to be precluded by the absence of functional or anatomical correlates that can be detected in utero, and the observed incomplete penetrance. The findings by LEGENDRE et al. [12] support the recommendations that genetic screening for known pathogenic variants, including those novel variants described in their study, be offered to patients with clinical features of familial IPF, onset before the age of 50 years, or extrapulmonary features of multi-system mendelian disorders that cause pulmonary fibrosis $[18,19]$. 
Two other striking findings in this study include the frequent absence of classical computed tomography (CT) scan indicators of definite UIP, and the frequent occurrence of lung cancer. The predominance of ground glass opacities and non-UIP CT patterns, which has been previously described in familial forms of IPF [20], suggests that the American Thoracic Society/European Respiratory Society/Japanese Respiratory Society/Latin American Thoracic Society criteria might not be applicable in patients with SFTPA mutations, or that this is a different disorder [3]. In fact, others have proposed that the label "idiopathic pulmonary fibrosis" fails to accurately describe the full spectrum of disease in patients with lung fibrosis, and that it might be renamed or sub-classified to incorporate the presumptive aetiology [21, 22]. Regarding the frequency of lung cancer in patients with SFTPA mutations [12, 13], the link between chronic epithelial stress and neoplasia has long been established [23], and patients with pulmonary fibrosis are at increased risk of developing lung cancer [24]. More studies like that contributed by LEGENDRE et al. [12], and complementary studies addressing the mechanisms by which familial IPF-associated variants lead to genotoxic stress in epithelial cells, will likely reveal biomarkers that define disease endotypes and molecular targets for preventive or disease-modifying therapies focused on the underlying aetiology of IPF and lung cancer.

Conflict of interest: A.S. Kristof has nothing to disclose.

Support statement: This work was supported by Canadian Institutes of Health research grant PJT 155971. Funding information for this article has been deposited with the Crossref Funder Registry.

\section{References}

1 Kropski JA, Blackwell TS. Progress in understanding and treating idiopathic pulmonary fibrosis. Annu Rev Med 2019; 70: 211-224.

2 Lederer DJ, Martinez FJ. Idiopathic pulmonary fibrosis. N Engl J Med 2018; 379: 797-798.

3 Raghu G, Remy-Jardin M, Myers JL, et al. Diagnosis of idiopathic pulmonary fibrosis. An Official ATS/ERS/JRS/ ALAT Clinical Practice Guideline. Am J Respir Crit Care Med 2018; 198: e44-e68.

4 Kristof AS, Petrof BJ, Hamid Q, et al. An official American Thoracic Society workshop report: translational research in rare respiratory diseases. Ann Am Thorac Soc 2017; 14: 1239-1247.

5 Spagnolo P, du Bois RM, Cottin V. Rare lung disease and orphan drug development. Lancet Respir Med 2013; 1: 479-487.

6 Development of new therapeutic drugs and biologics for rare diseases. In: Field MJ, Boat TF, eds. Rare Diseases and Orphan Products: Accelerating Research and Development. Washington, National Academies Press (US), 2010.

7 Collard HR, Bradford WZ, Cottin V, et al. A new era in idiopathic pulmonary fibrosis: considerations for future clinical trials. Eur Respir J 2015; 46: 243-249.

8 Mathai SK, Newton CA, Schwartz DA, et al. Pulmonary fibrosis in the era of stratified medicine. Thorax 2016; 71: $1154-1160$.

9 Molina-Molina M, Borie R. Clinical implications of telomere dysfunction in lung fibrosis. Curr Opin Pulm Med 2018; 24: 440-444.

10 Whitsett JA, Wert SE, Weaver TE. Alveolar surfactant homeostasis and the pathogenesis of pulmonary disease. Annu Rev Med 2010; 61: 105-119.

11 Garcia CK. Insights from human genetic studies of lung and organ fibrosis. J Clin Invest 2018; 128: 36-44.

12 Legendre M, Butt A, Borie R, et al. Functional assessment and phenotypic heterogeneity of SFTPA1 and SFTPA2 mutations in interstitial lung diseases and lung cancer. Eur Respir J 2020; 56: 2002806.

13 Wang Y, Kuan PJ, Xing C, et al. Genetic defects in surfactant protein A2 are associated with pulmonary fibrosis and lung cancer. Am J Hum Genet 2009; 84: 52-59.

14 Raj A, Rifkin SA, Andersen E, et al. Variability in gene expression underlies incomplete penetrance. Nature 2010; 463: 913-918.

15 Brownell R, Kaminski N, Woodruff PG, et al. Precision medicine: the new frontier in idiopathic pulmonary fibrosis. Am J Respir Crit Care Med 2016; 193: 1213-1218.

16 Nilsson EE, Sadler-Riggleman I, Skinner MK. Environmentally induced epigenetic transgenerational inheritance of disease. Environ Epigenet 2018; 4: dvy016.

17 Richards S, Aziz N, Bale S, et al. Standards and guidelines for the interpretation of sequence variants: a joint consensus recommendation of the American College of Medical Genetics and Genomics and the Association for Molecular Pathology. Genet Med 2015; 17: 405-424.

18 Spagnolo P, Cottin V. Genetics of idiopathic pulmonary fibrosis: from mechanistic pathways to personalised medicine. J Med Genet 2017; 54: 93-99.

19 Kropski JA, Young LR, Cogan JD, et al. Genetic evaluation and testing of patients and families with idiopathic pulmonary fibrosis. Am J Respir Crit Care Med 2017; 195: 1423-1428.

20 Lee HY, Seo JB, Steele MP, et al. High-resolution CT scan findings in familial interstitial pneumonia do not conform to those of idiopathic interstitial pneumonia. Chest 2012; 142: 1577-1583.

21 Wolters PJ, Blackwell TS, Eickelberg O, et al. Time for a change: is idiopathic pulmonary fibrosis still idiopathic and only fibrotic? Lancet Respir Med 2018; 6: 154-160.

22 Wells AU, Brown KK, Flaherty KR, et al. What's in a name? That which we call IPF, by any other name would act the same. Eur Respir J 2018; 51: 1800692.

23 Grivennikov SI, Greten FR, Karin M. Immunity, inflammation, and cancer. Cell 2010; 140: 883-899.

24 Tzouvelekis A, Gomatou G, Bouros E, et al. Common pathogenic mechanisms between idiopathic pulmonary fibrosis and lung cancer. Chest 2019; 156: 383-391. 